\title{
Re-make of Sheet Metal Parts of End of Life Vehicles - Research on Product Life-Cycle Management
}

\author{
George L. Kovács ${ }^{1,2}$ and Imre Paniti ${ }^{1}$ \\ ${ }^{1}$ Computer and Automation Research Institute, Hungarian Academy of Sciences \\ ${ }^{2}$ PMMIK, Pécs University of Sciences, Pécs, Hungary \\ \{kovacs.gyorgy, paniti.imre\}@sztaki.mta.hu
}

\begin{abstract}
Today it can be accepted as fact that mankind is running out and will soon have shortages of energy, row materials and water. Sustainable development (or more often used sustainability) means to use as little materials, energy and water as possible. Material saving causes energy and water saving as well. Product Life Cycle Management (PLCM) of any kind of product starts when the idea of a product is born and lasts until complete dismissal through steps as detailed design, assembly design, part manufacturing, assembly, testing, diagnostics and operation, advertisement, service, maintenance, etc. Then finally our topics, namely dismantling/ disassembly and dismissal are coming. Dismissal often can be substituted by re-cycling or re-use, or re-make.
\end{abstract}

Keywords: re-use, end of life vehicle, sheet-metal, incremental sheet forming, sustainability, product life-cycle.

\section{Some Definitions and Abbreviations}

The motivation of the example used during this study was born from the EU Directive 2000/53/EC [1a] according to which by 2015, the portion of each vehicle that should be recycled or reused have to increase to $95 \%$. To avoid being too abstract in the paper we use the management of sheet metal parts of worn-out or crashed cars as example. We show a possible way of value evaluation and measurement of values during PLCM. On the other hand we deal with the complexity and problems of decision making during processing of used sheet-metal parts' 1, if the main goal is Remake (reuse). The decisions are about dismantling and remake as e.g. re-cycling or re-use with or without repair, how to repair, etc.

Some definitions and abbreviations will be given first to help better understanding some expressions in the context of worn out or broken or simply End of Life (EOL) vehicles. Remake, re-use and re-cycling should not be mixed up with waste management, as our goal is to use everything instead of producing waste.

- ELV (End of Life Vehicle, EOL vehicle): cars after collision or worn out cars

- Shredder (S): a strong equipment breaking, tearing everything into small pieces, to shreds, almost like a mill. 
- Dismantling/disassembly (DM, DA): to take out parts for further manipulations, opposite to assembly.

- Delayed or conditional decision (DD, CD), a decision that depends on something, and will be done later on

- Dismissal/liquidation: to get rid of the whole or of certain parts using a shredder and then the rest is "buried" (disposed)

- Re-use: to use again certain parts after dismantling, as they are or applying correction (Re-make). Usage as second-hand parts.

- Re-cycle: melting the metals (and plastic) separately for getting useful raw material

- Sheet metal parts - we use it mainly to speak about car body parts.

- Dangerous material: materials, which have to be collected and managed separately in the case of every car. For example chemicals in the battery, or in the air conditioner, oil, gasoline, etc.

- ISF: Incremental sheet forming: a technique to form requested sheets by a series of small incremental deformations based on corresponding CAD/CAM information.

- CAD/CAM (Computer Aided Design/Computer Aided Manufacturing) data: digital information, describing car body sheet metal parts: gained from original design, or from 3D measurements, etc. It can be used to produce $\mathrm{CNC}$ code

- CAD/CAM information of car body sheet metal parts for correction of shape errors. Data scanned from the part to be corrected vs. data of the needed sheet.

- Thickness measurement: the most important information to qualify the sheet

- 3D shape measurement (scanning): to measure the actual shape (in most cases with laser technology), and to produce CAD/CAM data for comparison.

- Scanning: optical (laser) way of 3D shape management, resulting in a cloud of surface data, describing the shape, input for CAD/CAM and CNC,

- Decisions at several levels: they may be Automatic, Semi-automatic, or Human (DA, DSA, DH)

- Decision assisting tools: optical and other measurements, AI programs, human considerations

- Product Life-Cycle-Management (PLCM): management of any kind of product for its whole life-cycle. Almost everything can be taken into consideration as a product.

\section{Introduction and State-of-the-Art}

Management of EOV (EOL vehicles) is a rather complicated task, which needs to process several technical and legal steps (paperwork). All parts of all vehicles have to be under control during their whole Life-Cycle, including permissions to run, licence plates, permissions to stop, informing local authorities, etc. In this paper we deal only with technical aspects of a restricted set of parts, namely with sheet-metal parts. 


\subsection{Some Sources (for Example [1]) Simplify the Procedure to the Following 3 Steps:}

- Depollution; Dismantling; Shredding

The main goal is achieved, but several points (decision points) remain open and several questions unanswered, and nobody really knows what to do and how to do and what are the consequences of certain activities. What happens with the different parts?? Is there anything worth repairing, etc. ?

There are others, who claim that the process is a little more complicated. The following EU suggestion does not go into details; it is a straightforward average procedure including paper work, which is crucial if someone deals with EOV.

\subsection{According to [2] the Procedure of Dismantling Should Be the Following}

- Delivery of EOL vehicle

- Papers and documents are fixed, permissions issued (or checked if issued by others)

- Put the car onto a dry bed: remove dangerous materials and liquids, store everything professionally

- Select useful parts, take them out and store under roof

- Sell/offer for selling the tested parts

- Press the body to help economic delivery

- Re-use raw materials

A little more precise description of the removal sequence of different important parts/materials with more details, however still without decision points is the following:

- Remove battery and tanks filled with liquid gas

- Remove explosive cartridges of airbag and safety bells

- Remove gasoline, oils, lubricants, cooling liquid, anti- freezing, break liquid, airconditioner liquid

- Most careful removal of parts containing quick silver

- Remove catalysts

- Remove metal parts containing copper, aluminium, magnesium

- Remove tyres and bigger size plastic parts (fender/bumper, panel, containers for liquids)

- Remove windshields and all glass products

\subsection{The IDIS Web Site [3] has the Following Opinion}

The International Dismantling Information System (IDIS) was developed by the automotive industry to meet the legal obligations of the EU End of Life Vehicle (ELV) directive and has been improved to an information system with vehicle manufacturer compiled information for treatment operators to promote the environmental treatment 
of End-of-Life-Vehicles, safely and economically. The system development and improvement is supervised and controlled by the IDIS2 Consortium formed by automotive manufacturers from Europe, Japan, Malaysia, Korea and the USA, covering currently 1747 different models and variants from 69 car brands.

The access to and the use of the system is free of charge. The basic steps of dismantling suggested by IDIS 2 are as follows:

- Batteries -- Pyrotechnics -- Fuels

- AC( Air Conditioner) - Draining - Catalysts

- Controlled Parts to be removed - Tires -- Other Pre-treatment

- Dismantling

\subsection{At GAZ Autoschool [4] in the UK the Following Are Underlined as the Most Important Steps to Follow}

1. Removing vehicle doors, bonnet, boot, hatch. Removing these items early in the dismantling process enables easier access to vehicle interior, reduces restriction in work bays and minimises the risk of accident damage to potentially valuable components.

2. Removing interior panels, trim, fittings and components. This is a relatively clean and safe operation which maximises the resale opportunities available for items whose value depends on appearance/condition and which may be damaged if left on the vehicle. Components to be removed include dashboard, instrument panel, heater element, control stalks, steering column.

3. Remove light clusters: An easy process but one which needs care to avoid damage. Once removed items need to be labeled and stored to enable potential re-sale.

4. Removal of wiring harness: The harness should be removed without damage, meaning that all electrical components are unclipped and the wires pulled back through into the interior of the car so that it can be removed complete and intact. Harness should be labeled and then stored appropriately.

5. Removal of Engine and Gearbox: This will involve the use of an engine hoist, trolley jacks and axle stands, and will often necessitate working under the vehicle for a short period to remove gear linkages etc. Often the dirtiest and most physical task. Engine and gearbox oil together with engine coolant will need to be drained and collected for storage.

6. Engine dismantling: Engines are kept for resale where possible.

7. Gearbox dismantling: Gearboxes are kept for resale where possible.

8. Brakes and shock absorbers: Brake components are checked and offered for resale where they are serviceable.

\subsection{Finally We Refer to [5], Which Is a Survey and Case Study on Serial Flow Car Disassembly}

The main considerations and results of the survey are the following. 


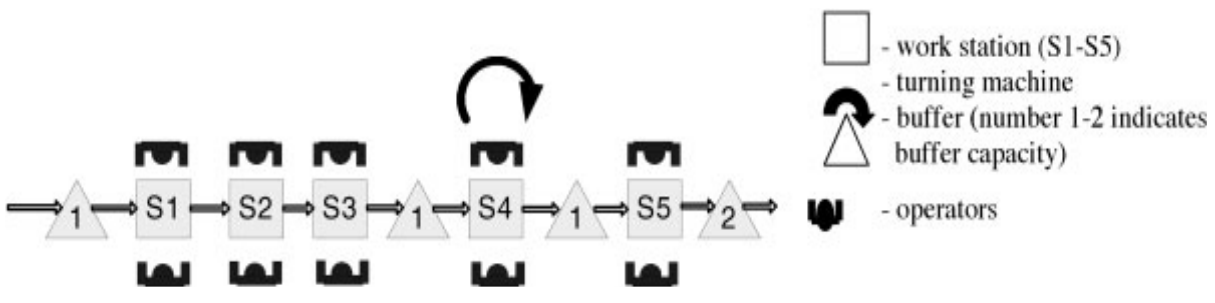

Fig. 1. Serial-Flow Car Disassembly

- In the European Union over 15 million passenger cars were sold in 2004 according to European Automobile Manufacturers Association (ACEA, n.d.), up 1.7\% from 2003.

- Around 8 million end-of-life vehicles (ELVs) are disposed of annually in the EU (ACEA, 2004) and this number is expected to increase.

- An EU directive (2000/53/EU; European Parliament, 2000) [1a] requires that wastes from ELVs must be reduced to $15 \%$ of the total car weight by the year 2006 and to $5 \%$ by 2015 .

The suggested technology can be represented by Fig. 1., where one can see, that the system used five stations and four buffers.

- At stations 1-3 glass, rubber, and interior are removed.

- At station 4 the "turning machine" rotated cars upside down to facilitate engine and gearbox unfastening

- At station 5 the engine and gearbox are removed.

The procedure is the following:

1. Take a lot of pictures before you begin the disassembly process, including pictures of the interior. This important issue is rarely mentioned by other authors

2. Get a box of zip lock plastic bags in each size available to store every nut, bolt, hinge, clip, shim, etc. Make color marks to all.

3. Make sure you have a pen and a notebook by your side at all times to document any helpful reminders, parts in need of replacement and to take inventory

4. Remove the fenders, hood and trunk lid with the assistance of at least one able body to avoid damage and personal injury

5. Remove the front windshield and the rear window by first removing the chrome molding from the outside of the car, being careful not to scratch the glass.

6. This would be a good point to gut the interior. Remove the seats, doors and interior panels, carpeting and headliner.

7. Clear the firewall and take all the accessories off the engine.

8. Go through your notebook and highlight all the parts that need to be replaced and make a separate "to do" list for ordering them.

The most characteristic in the above study is a practical approach: be very careful and document everything, use bags and color pens and notebook to keep track of all parts 
and activities. This non-stop bookkeeping may hinder effective and fast work, however surely helps in knowing and tracing everything, if needed.

\section{Sheet-Metal Parts' Management}

It can be seen from the previous examples that practically nobody deals with the special problems of sheet metal parts of EOL vehicles. On the other hand it is clear that sheet metal parts are only a certain percentage of an EOL vehicle. But it is clear, that almost every EOL vehicle has several sheet metal parts, which could be re-used with or without corrections, with or without re-paint. This makes us to believe that it is worthwhile to deal with sheet metal parts separately, moreover in the following part of our study this will be our only issue. To think and to speak about re-use (re-make, re-shape) as a practical issue, we need as minimum:

(a) proper dismantling technology to remove sheet-metal parts without causing damages to them

(b) a measurement technology to evaluate the dismantled part and a software to compare the measured values to requested values, to define whether the dismantled part is appropriate or needs correction and to decide its applicability to be used for another vehicle, and finally

(c) a technology to correct slightly damaged sheets, based on CAD/CAM information. This information may come from design (new or requested parts) or through measurements by a scanner (actual, dismantled part).

Our staff using ISF technology and the robotic laboratory of SZTAKI is able to perform the requested operations on our machines and on the software

\subsection{A Sheet-Metal Decision Sequence}

Our approach needs to follow a rather complicated decision sequence; it will be detailed only for sheet metal (SM) parts, supposing the available Incremental Sheet Forming (ISF) facilities.

It is the following, using the above defined abbreviations, emphasizing decision types and points:

1. Car arrives: on wheels or on a trailer, papers and documents are fixed, permission issued (or checked if issued by others)

2. It goes or it is taken to the dismantling bed (dry bed)

3. Remove liquids and dangerous materials (Unconditional)

4. Decision 1: Shredder (S) or dismantling (DM) or delayed decision after the beginning of dismantling (DD). Decision is done basically by human, eventually assisted by measurements, or even by 3D part modeling

(a) if S: no more work to do: car goes to shredder and then burial (dismissal)

(b) if DM: disassembly starts, parts are taken off one by one until the last one, based on a given protocol for all car types. 
(c) if DD: disassembly starts, parts are taken off one by one, and sorted and stored, until the next decision can be done

5. Decision 2: S or DM - done at any time. Decision may be partial shredder (PS) and partial dismantling (PD) after a while.

(a) if PS\&PD: certain parts are taken apart, the rest goes to shredder.

(b) if DM or DD and PD are done, now we have a lot of parts organised somehow

6. Decision 3: Select sheet metal parts: automatically or manually or hybrid way, keep SMs, put away the rest

(a) Examine all SM parts, first thickness (TH) measurement is done

(b) if $\mathrm{TH}$ is too small, part goes to $\mathrm{S}$. The rest goes to border measurement (SB), as borders (SB), of a sheet may easily be damaged during dismantling.

(c) SB may be done by optics and AI and/or by human or both, after each other.

(d) if SB is repairable or good, make shape measurement (SHM)

(e) Compare measured sheet (MS) to standard shape ( $\mathrm{SH}$ ). $\mathrm{SH}$ can be taken by measuring a failure-free sample, or from any appropriate catalogue. For processing we need CAD data in both cases.

(f) Compare $\mathrm{SH}$ with MS. and calculate differences somehow, it is the deviation from standard (DS)

7. Decision 4: if DS is small enough (defined by the customer, who will need the part, or it is an average value generally accepted), part goes to repair. The rest goes to shredder.

8. Decision 5: repair by hand, by ISF or combined, any sequence is possible

(a) if ISF: part goes to ISF centre together with its CAD/CAM code, and processed

(b) if Manual or Combined: part goes to worker, when needed, after or before ISF

(c) if ISF is done - a final measurement is needed (SHM).

9. Decision 6: if accepted it goes to the shop or to a workshop for painting, and then to a shop.

(a) if rejected it goes back to 6.6.

10. The part is accepted, sent to the shop or to business again

This decision sequence - naturally - can be taken into account as a small and timely short period of the PLMC, namely of the EOL cars' sheet-metal parts. Each move and activity have certain actual prices, which are commonly accepted, however we know that they are not really correct, they do not support sustainability, and on the other hand often increase negative effects.

\subsection{Evaluation of Costs and Advantages of the Re-use and Re-make of Sheet- Metal Parts of EOVs}

After such a long, or long-looking decision procedure we need a methodology for evaluation of all, what we do or do not perform to have re-usable sheet-metal parts from parts of EOL vehicles.

The simplest way would be simply compare costs and prices of all involved parts (good, to be corrected, etc.) and services (scanning, ISF, manual work, painting, etc.) and all conditions (shredder or repair, etc.). 
Today that is the only way some people follow, if any. Generally a very fast view at the EOV is enough to send it to the shredder, as this is the simplest decision with the smallest risk. To be more precise, the risk is there, but rather hidden.

The cost/value estimations and comparisons can be performed relatively easily, however the results correspond only to the present economical - political situation and to the actual financial circumstances, and would not say anything about the future, what is embedded into the "sustainability", "footprint" and "side-effects" (see later on). We believe that there exist giving appropriate tools and means for "real", "future centric" evaluations, thus we need to find and use them.

Our choice are the KILT model and the TYPUS metrics, which will be explained and used for our study, for details see [6], [7], [8] and [8a].

The main goal of the above tools is to model and quantify the complete delivery (all products, side-products, trash and effects of them, i.e. all results) of a firm, and to model all interesting and relevant steps of the LC (or LCM). It is clear from the definitions (see later and the references) that any production steps can be evaluated and can be understood as cost values. If we speak about car production, the input is row material, machining equipment and design information, and people, who work, etc. Output (delivery) is the car. Sheet metal production is a little part of car making, generally prior to body assembly. For our study we take into consideration only sheetmetal parts.

We consider and make measurements, comparisons, re-make by using ISF and repaint, and other actions. These can hardly be compared with the "simple" processes used in new car manufacturing. Every step of the decision sequence below can be investigated one by one, taking into account all effects and side-effects. For the sake of simplicity only the input (sheet metal part to be measured and perhaps corrected) and the output (sheet-metal part ready to be used again) may be enough.

Fig. 2. shows some qualitative relationships, which cannot be avoided if environmental issues, sustainability, re-use and our future are important points.

Fig. 2 gives a general picture of our main ideas, and it needs some explanation. See [8a] for more details. It is a rather simplified view of some main players in the production/service arena, however it still shows quite well certain main qualitative relationships. We believe that these can be used to understand what is going on in our (engineering-manufacturing-sustainable) world. 


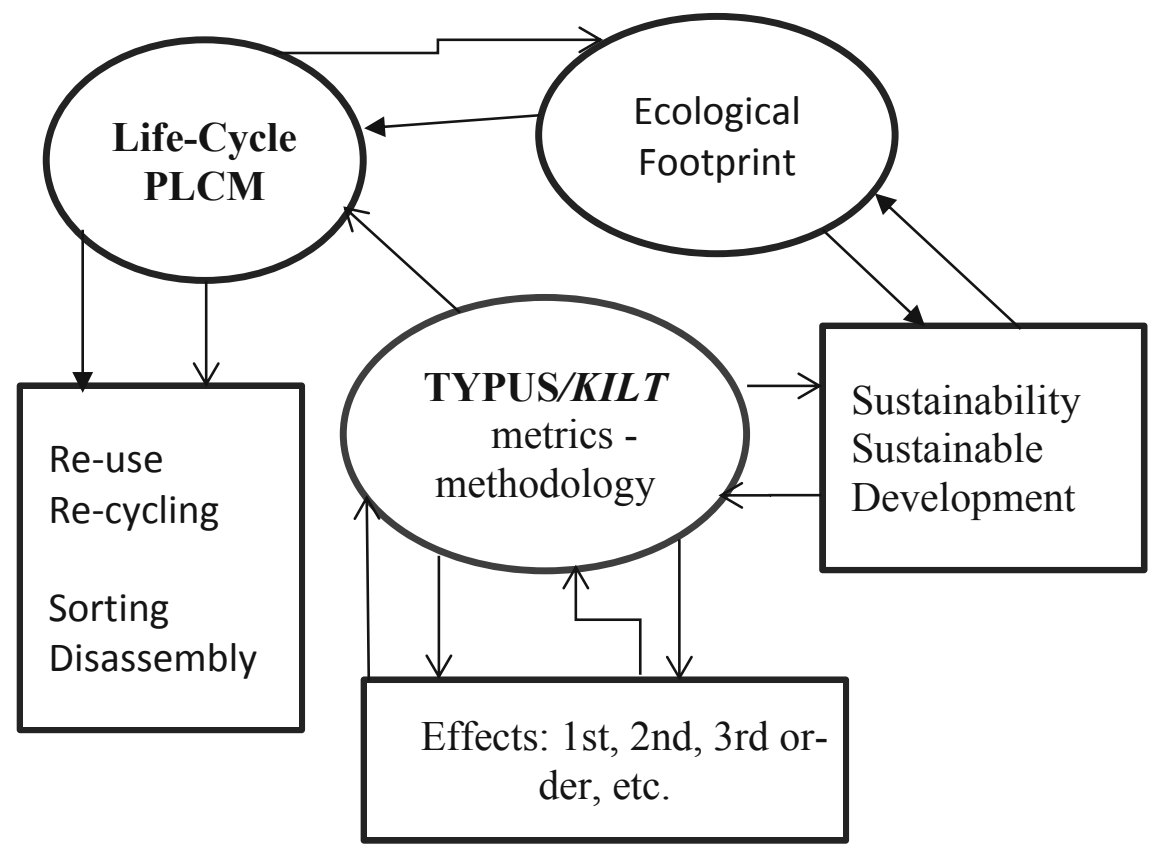

Fig. 2. Re-use, PLCM, ecology, sustainability and KILT

The TYPUS/KILT metrics, methodology and model give us a possibility to better understand and evaluate production results and their components in terms of the K, I, L, $\mathrm{T}$ values. They give us a method of calculations and comparisons based on realistic values. The side effects and 2 nd and 3rd order effects, etc. mean the following: let us consider a simple and simplified example: to produce a hybrid car (today) means (among others) to produce and build in two engines, two engines need more metal than one (side effect), to produce more metal we need more electrical energy and more ores ( 2 nd order side effect), to produce more electricity more fuel is necessary and to produce more ores needs more miners' work (3rd order side effect), etc., and it could be continued. It is a hard task to know how deep and how broad we should go with such calculations. And if we take a look at our example there are several other viewpoints that could be taken into account. Just one example: the increased water consumption during mining. We have to confess that in the recent study on sheet-metal parts of EOL cars we do not deal with the side-effects at all. The reason is simply that we are in the beginning of the research and only try to define what should we do in this aspect.

Today the whole world, all at least most countries understand the importance of natural resources, environment, and based on this understanding reuse and recycling are getting more and more important in everyday life, as well as the decrease of CO2 emission, etc. These all request to keep energy, water, natural resources, manpower, etc. consumption in a moderate, sustainable level. This leads to sustainable development, or even to sustainability. 


\subsection{The KILT Model and the TYPUS Metrics}

Just to remember we repeat some main points of the KILT model and the TYPUS, which are properly explained in [6,7,8 and 8a].TYPUS metrics means Tangibles Yield per Unit of Service. It is measured in money - on ecological basis. It reflects the total energy and material consumption of (all) (extended) products of a given unit, e. g. of an enterprise. But it can be applied for bigger (e.g. virtual enterprises) or any smaller units (e.g. workshop or one machine) or for any selected actions (e.g painting, bending, cutting, etc.) of any complexity. In this study it is all only about sheet-metal management of EOV, however due to the complexity of the problems and due to the status of the research we still do not make real value calculations.

The metrics assumes several things, as : life-cycle function; material and energy provisions during manufacturing, operation, repair, reuse or dismissal, etc.

\subsubsection{KILT is an arbitrarily, but properly chosen implementation of TYPUS,} we could imagine other realizations as well, However, recently the given definitions seem to be the best to manage the requested goals, as far as the authors believe. The related TYPUS metrics is further discussed later on. In earlier models and considerations The delivered quantities (all outputs), Q, is assumed to depend on the contributed financial ( I ) and human ( $\mathbf{L}$ ) capitals, plus the know-how ( K ) (innovation) and the tangibles ( $\mathbf{T}$ ) have non negligible effects..

The relationships are still work as multiplication and looks the next:

$$
\mathrm{Q}=\mathrm{f}(\mathrm{K}, \mathrm{I}, \mathrm{L}, \mathrm{T})
$$

Summarizing the different factors we get some content to all of them as capital, knowledge, activity, material, etc. at the same time:

K: Technical capital - knowledge, technology, know how, etc.- intangibles

I: Financial capital- investment, capital, etc.

L: Human capital- labor, traditional labor, human efforts, welfare charges, etc.

T: Natural capital - tangible resources: material, consumables, ecologic fees, utilities, commodities, etc.

All the contributed technical K, financial I, human L and natural $\mathrm{T}$ capitals are included, and there is a tetra-linear dependence, which assumes to operate nearby equilibrium assets. The KILT models reliably describe the delivered product quantities, Q. Lacking one contribution (any of the above factors has a value of 0), the balance is lame, and the reckoned productivity figures, untruthful or meaningless.

The tetra-linear dependence means the equivalence of assets alone, and their synergic cumulated action. The company return is optimal, when the (scaled) factors are balanced; the current scaling expresses in money the four capitals (the comparison of non-homogeneous quantities is meaningless; the output $\mathrm{Q}$ has proper value, with the four inputs homogeneity). The return vanishes or becomes loss, if one contribution disappears. The loss represents the imbalance between constituent (know-how, money, work out-sourcing, bought semi-finished parts, etc.) flows. 


\subsubsection{The TYPUS Metrics}

TYPUS, tangibles yield per unit service: the measurement plot covers the materials supply chain, from procurement, to recovery, so that every enjoyed product-service has associated eco-figures, assembling the resources consumption and the induced falls-off requiring remediation. The results are expressed in money. The point is left open, but, it needs to be detailed, to provide quantitative (legal metrology driven) assessment of the "deposit-refund" balance.

The metrics is an effective standard, aiming at the natural capital intensive exploitation. The supply chain lifecycle visibility needs monitoring and recording the joint economic/ecologic issues, giving quantitative assessment of all input/output materials and energy flows.

We have to apply these considerations to the decision sequence of "remake - no remake" of sheet metal parts of EOV, taking into account all technological steps and human actions including their side effects, if we can understand, define, measure and quantify them. It will take some ore time and a lot of researchers' efforts. To be a little positive we are convinced that the above discussed metrics and model can be used between any two points of the PLC, i.e. all costs, outputs, results, effects and side effects can be measured, calculated and evaluated in the decision sequence of sheet metal parts of EOL cars in our case.

\section{Some Technological Issues of ISF}

There are several open issues concerning the ISF technology and 3D measurements. We have to make several experiments with the scanner system to have an exact view of the measured sheet, and with the software systems, which compare the different surfaces with each other and with the accepted shape's data. These may be results of scanning, but more often results from the design (CA/CAM) processes. Finally the software dictates - and the humans generally accept - what should be done using ISF. On the ISF side we still have problems with accurate shape and thickness measurements. These works are running recently with high efforts. Fig. 3 presents an ISF experiment with an industrial robot using a $50 \mathrm{~cm}$ x $50 \mathrm{~cm}$ frame for the sheet. For car parts we can use the same robot, but a larger, and different frame will be needed. It is on the design table already.

From the technological point of view, the ISF consists of the gradual plastic deformation of a metal sheet by the action of a spherical forming tool whose trajectory is numerically controlled. The interest in evolution of ISF is rather old; it started in 1967 with the patent of Leszak [10]. This idea and technology is still active today in the field of producing sheet metal and polystirol parts in small batch and one-of-akind production, rapid prototypes, in medical aid manufacturing and in architectural design. A specific forming tool is mounted on the machine spindle or on a robot, and it is moved according to a well-defined tool path to form the sheet into the desired shape. Several ISF strategies have been developed which mainly differ in equipment and forming procedure. In particular, the process can be divided into: 


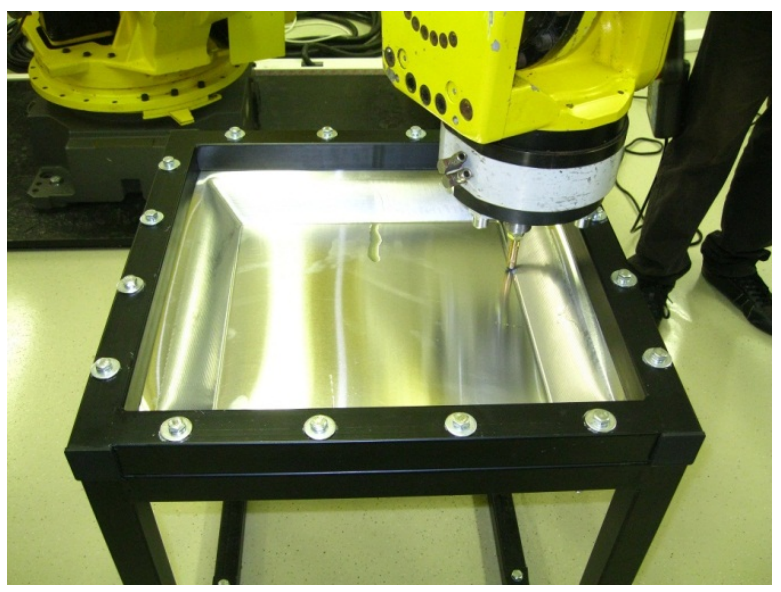

Fig. 3. ISF experiment with a FANUC robot

\section{- Single Point Incremental Forming (SPIF)}

Here the sheet metal is shaped by a single tool (with a faceplate supporting the initial level of the sheet).

\section{- Two Points Incremental Forming (TPIF)}

where the sheet metal shaping is ensured by: a) two counter tools or b) a local die support that is a sort of partial / full die.

In Full Die Incremental Forming the tool shapes the sheet alongside a die; this die could be produced from cheap materials such as wood, resin or low cost steel; the use of a die ensures a better and more precise shape of the final piece.

As repair tool only SPIF or TPIF with synchronised counter tools can be considered because the manufacturing of full or partial dies needs more time and money. On the other hand SPIF has some drawbacks compared to TPIF with two counter tools.

Experimental Investigations and Numerical Analysis were carried out by Shigekazu Tanaka et al. [11] to examine the residual stress in sheet metal parts obtained by incremental forming operations because distortion were observed after removing the outer portion of the incremental formed sheet metal part. Results showed that "tension residual stress is produced in the upper layer of the sheet and compression stress in the lower", furthermore the stress is increasing with the increase of the tool diameter [11].

Crina Radu [12] analysed the correlation between the accuracy of parts processed by SPIF using different values of process parameters and the distribution of the residual stresses induced in the sheets as results of cold incremental forming.

The hole drilling strain gauge method was applied to determine the residual stresses distribution through the sheet thickness. Experiments showed that the increase of tool diameter and incremental step depth increased residual stresses, which led to higher geometrical deviations [12]. 
J. Zettler et al. stated in their work that SPIF indicate "great residual stresses to sheet during the forming which lead to geometrical deviations after releasing the fixation of the sheet". They introduced a spring back compensation procedure in which an optical measurement system is used for measuring the part geometry after the forming [13].

Silva [14] et al. made some Experimental Investigations and Numerical Analysis to evaluate the applicability and accuracy of their analytical framework for SPIF of metal sheets. They stated that "plastic deformation occurs only in the small radial slice of the component being formed under the tool. The surrounding material experiences elastic deformation and, therefore, it is subject of considerably lower stresses."

In order to compensate springback more effectively (in-process) online residual stress measurements are suggested. Residual Stress Measurement Methods can be characterized according to the length detection over the stresses balance.

Feasible Non-Destructive Testing (NDT) Methods based on a summary of Withers et al. [15] are Ultrasonic and Magnetic Barkhausen noise (MBN) measurements. By comparing these methods we can say that ultrasonic solutions can be used for nonferromagnetic materials too, but for the evaluation of Multiple Residual Stress Components the Barkhausen noise (BN) testing is preferable. The work of Steven Andrew White showed that "BN testing is capable of providing near-surface estimates of axial and hoop stresses in feeder piping, and could likely be adapted for in situ feeder pipe inspection or quality assurance of stress relief during manufacture" [16].

By adapting the MBN solution of Steven Andrew White to Incremental Forming of sheet metals we can realize an enhanced concept of J. Zettler et al. [13] where the optical measurement system is replaced/extended by a MBN measurement device integrated into a forming tool. This solution may allow finishing the manufacturing/repairing of a part with high geometrical accuracy however, without releasing the fixation.

\section{Conclusions and Further Plans}

Our real goal is to give some means and tools to calculate different values which correspond to different phases of the life-cycle of a product (PLC). We specially emphasize re-use and re-cycling as important LC phases, due to the approaching water-, energy- and raw material-shortages. Generally on product we mean anything which is used by simple users (a car, a cup, a bike, or a part of them, etc.), or which are used by dedicated users to produce or manage other products (a machine tool, a robot, a house, a test environment, etc.), or which are used to manage everything else (a firm, a factory, a ministry, etc.). We differentiate between simple products and extended products (as traditional and extended enterprise) and between tangible and intangible parts (aspects) and service is taken into account as a product, too.

In the recent study we restrict ourselves to a very narrow part of the PLCM of cars: to evaluate EOL cars' sheet-metal parts, then to decide whether to re-make (repair, reuse) them or let them go to the shredder to be dismissed. 
During our research to assist re-use of sheet metal parts we had several problems to solve to make waste as little as possible, and to prefer re-use, or re-make. There were several machine- and human decisions, which need support. An important assistance is $3 \mathrm{D}$ modelling and visualisation to help human decision making if a simple view is not enough, as it cannot be exact enough. The set and methods of decision making drove us to the way of cognitive info-communication. This way should be extended and explained in more details in the future.

We showed that the above explained simple multiplications forms of KILT cannot yet be used for economically useful calculations, they contain only several ideas and qualitative relationships to go on a right way. We plan to find proper relationships to use our ideas and formulae for real world situations to assist not only designers and engineers in their work, but politicians and other decision makers as well. These studies and their resulting calculations, values and suggestions how to proceed will be in a following study. Specific applications to ISF technology may mean simplifications and easier understanding and using of the metrics and the model.

\section{References}

1. Gaillot, O., McCormack, I.: Depollution and Shredder Trial on End of Life Vehicles in Ireland, RPS Consulting Engineers, West Pier, Business Campus, Dun Laoghaire, Co., Dublin, Ireland (study)

1a. Directive 2000/53/EC of the European Parliament and of the Council of 18 September 2000 on end-of-life vehicles, http://www.municipalwasteeurope .eu/ summary-current-eu-waste-legislation

2. http://www. autobonto.eu/bontas.html

3. http://www. IDIS 2 . com

4. http://www.gazautoschool.org.uk/home-2/vehicle-dismantling

5. Kazmierczak, K., Patrick, W., Winkel, J., Parkallé, L.: A Case Study of Serial-Flow Car Disassembly: Ergonomics, Productivity and Potential System Performance. Human Factors and Ergonomics in Manufacturing 17(4), 331-351 (2007), http://onlinelibrary.wiley.com/doi/10.1002/hfm.20078/pdf

6. Michelini, R.C., Kovács, G.L.: Integrated Design for Sustainability: Intelligence for EcoConsistent Products and Services. In: EBS REVIEW, Innovation, Knowledge, Marketing and Ethics, pp. 81-94 (Winter 2002/2003) ISSN 1406-0264

7. Michelini, R.R.: Knowledge Enterpreneurship and Sustainable Growth, 325 pages. NOVA Science Publ. (2008)

8. Michelini, R.R.: Knowledge Society Engineering, A Sustainable Growth Pledge, 350 pages. NOVA Science Publ. (2010)

8a. Kovács, G.: Understanding enterprize delivery using the TYPUS metrics and the KILT model. In: Proc. of CSIT 2011, Garmisch, Germany, September 27-October 02, pp. 1-7 (2011)

9. Dernbach, J.C. (ed.): Stumbling towards sustainability. The Environmental Law Institute, Washington, D.C., pp. 1-968 (July 2002) ISBN: 1-58576-036-6

10. Leszak, E.: Apparatus and Process for Incremental Dieless Forming. US Patent 3342051A1 (September 19, 1967) 
11. Tanaka, S., Nakamura, T., Hayakawa, K., Nakamura, H., Motomura, K.: Residual Stress in Sheet Metal Parts Made by Incremental Forming Process. In: Proceedings of the 9th International Conference on Numerical Methods in Industrial Forming Processes, Porto, Portugal, June 17-21, pp. 775-780 (2007), http://dx.doi.org/10.1063/ 1. 2740904

12. Radu, C.: Analysis of the Correlation Accuracy-Distribution of Residual Stresses in the Case of Parts Processed by SPIF. In: Mathematical Models and Methods in Modern Science - 14th WSEAS International Conference on Mathematical Methods, Computational Techniques and Intelligent Systems (MAMECTIS 2012), Porto, Portugal, July 1-3, pp. 195-199 (2012)

13. Zettler, J., Rezai, H., Hirt, G.: Springback Compensation for Incremental Sheet Metal Forming Applications, 7. LS-DYNA Anwenderforum, Bamberg (2008),

http: / / www . dynamore.de/de/download/papers / forum08/dokumente/ C-I-03.pdf/view

14. Silva, M.B., Skjoedt, M., Bay, N., Martins, P.A.F.: Revisiting single-point incremental forming and formability/failure diagrams by means of finite elements and experimentation. Journal of Strain Analysis for Engineering Design 44(4), 221-234 (2009), doi:10.1243/03093247JSA522

15. Withers, P.J., Bhadeshia, H.K.D.H.: Residual stress part 2-nature and origins. Materials Science and Technology 17, 366-375 (2001)

16. http://www . phispace. net/spiffyprod/COGfeedworkshop2009.pdf 